\section{AhR in SLE}

Impaired recognition and clearance of cellular debris are important factors in the pathogenesis of systemic lupus erythematosus (SLE). A new study sheds light on the mechanisms that regulate immune tolerance to cell debris, suggesting a key role for the transcription factor AhR. "Cell death can have a profound impact on immunity," states Tracy McGaha. "When cells become apoptotic they can drive immune-suppressive mechanisms that prevent immune reactivity against dying cell-associated antigens. This process is believed to be a key mechanism in the prevention of autoimmunity."

By studying changes in gene expression in murine phagocytes co-cultured with apoptotic cells, the researchers identified upregulation of AhR-responsive genes following exposure to cell debris. AhR specifically interacted with promoters to upregulate genes encoding cytokines such as IL-10; these changes induced a shift in phagocytes exposed to apoptotic cell debris towards an immunoregulatory phenotype. Blocking AhR led to reduced IL-10 production and a shift towards the production of pro-inflammatory cytokines, suggesting that AhR functions as a regulator of immune tolerance by suppressing inflammatory responses to cellular debris.

Using bone marrow-derived macrophages from Toll-like receptor 9 (Tlr9)-deficient mice, McGaha and colleagues revealed that the recognition of DNA from apoptotic cells by TLR9 can cause AhR activation and the production of IL-10. In two different mouse models of spontaneous lupus-like disease, pharmacological manipulation of AhR activity altered disease severity and progression. Blocking AhR activity increased the severity of lupus-like disease, whereas an AhR agonist ameliorated lupus-like disease. Interestingly, apoptotic cells also activated AhR and induced IL-10 production in human myeloid cells, and AhR transcriptional signatures similar to those seen in lupus-prone mice were seen in myeloid cells from patients with SLE. "Targeting AhR in human disease may have a similar therapeutic effect on disease activity to that seen in mice," concludes McGaha.

Joanna Collison, Associate Editor, Nature Reviews Rheumatology

This article is modified from the original in Nat. Rev. Rheumatol. (https://doi.org/10.1038/s41584-018-0024-6).

ORIGINAL ARTICLE Shinde, R. et al. Apoptotic cell-induced AhR activity is required for immunological tolerance and suppression of systemic lupus erythematosus in mice and humans. Nat. Immunol. https://doi.org/10.1038/s41590-018-0107-1 (2018)

\title{
AUTOSOMAL DOMINANT POLYCYSTIC KIDNEY DISEASE
}

\section{DNAJB11: another player in ADPKD}

Autosomal dominant polycystic kidney disease (ADPKD) is the most common monogenic form of kidney disease. Most cases result from mutations in $P K D 1$ and $P K D 2$, which encode the cilia membrane glycoproteins PC1 and PC2, respectively. A third ADPKD gene, GANAB, was described 2 years ago; however, the cause of ADPKD remains unresolved for $\sim 7 \%$ of families.

Now, use of whole-exome sequencing (WES) and next-generation sequencing (NGS) has identified an additional ADPKD gene, DNAJB11, which encodes a co-chaperone protein involved in endoplasmic reticulum (ER) protein processing. "This study demonstrates that ADPKD is more complex than previously thought, with a number of rare genes that phenocopy ADPKD," explains Peter Harris. "It also suggests that chaperone treatment might be valuable in some patients with $P K D 1$ and $P K D 2$ mutations that cause problems with protein folding and transport."

To identify ADPKD candidate genes, Harris and colleagues performed WES in two ADPKD-affected families, identifying DNAJB11. They then performed targeted
NGS of 65 candidate genes in a further 591 genetically unresolved families, leading to the identification of DNAJB11 mutants in five additional families. The affected patients were phenotypically similar, with small renal cysts and non-enlarged kidneys and liver cysts in some individuals. Chronic interstitial fibrosis, characteristic of autosomal dominant tubulointerstitial disease (ADTKD), was also evident. "The phenotypic overlap between ADPKD and ADTKD indicates the related pathogenic mechanisms," notes Harris. Characterization of the DNAJB11 variants suggested a role in the appropriate trafficking of $\mathrm{PC} 1$ and uromodulin, proteins involved in ADPKD and ADTKD, respectively. "These findings emphasize the involvement of ER quality control proteins in ADPKD and polycystic liver disease, due to the susceptibility of PC1 and ADTKD proteins such as uromodulin to trafficking deficiencies," says Harris.

Susan J. Allison

ORIGINAL ARTICLE Cornec-Le Gall, E. et al. Monoallelic mutations to DNAJB11 cause atypical autosomal-dominant polycystic kidney disease. Am.J. Hum. Genet. 102, 832-844 (2018)

\section{KIDNEY CANCER}

\section{Altered ammonia metabolism in ccRCC}

Metabolic abnormalities are a characteristic feature of clear cell renal cell carcinoma (ccRCC). A recent study by Celeste Simon and colleagues reports a role of altered ammonia metabolism in ccRCC progression. Ammonia is metabolized via the urea cycle, which converts free ammonia into urea.

"We have a long-standing interest in the relationship between oxygen availability, hypoxia-inducible factors, metabolism and tumour progression, and ccRCC represents an ideal platform to study this relationship," says Simon. "Moreover, we think that finding metabolic commonalities across tumours with diverse genetic backgrounds could significantly advance treatment efforts."

Using a combination of metabolomic, genomic and transcriptomic analyses, the researchers show that the expression of key urea cycle enzymes, including arginase 2 (ARG2) and argininosuccinate synthase 1 (ASS1), is strongly repressed in ccRCC tumours compared with healthy kidney tissue. In addition, re-expression of ARG2 or ASS1 in ccRCC xenograft models reduced tumour growth, indicating tumour-suppressive effects of these enzymes. Further analyses demonstrated that $A R G 2$ suppresses ccRCC growth via depletion of the essential coenzyme pyridoxal 5'-phosphate and via synthesis of polyamines, resulting in accumulations to toxic levels. As ccRCC cells are arginine auxotrophs owing to loss of urea cycle enzymes, the researchers also suggest that competition with tumourinfiltrating cytotoxic T cells for circulating arginine might have immunosuppressive effects.

"Changes in urea cycle enzymes in ccRCC protect a critical biosynthetic cofactor, avoid a toxic build-up of polyamines and potentially provide an immunosuppressive microenvironment," concludes Simon. "We now plan to determine if competition for arginine does indeed affect infiltrating cytotoxic $T$ cells and if there is a way to re-express urea cycle enzymes in ccRCC using epigenetic modifiers."

Ellen F. Carney

ORIGINAL ARTICLE Ochocki, J. D. et al. Arginase

suppresses renal carcinoma progression via biosynthetic cofactor pyridoxal phosphate depletion and increased polyamine toxicity. Cell Metab. https://doi.org/10.1016/ j.cmet.2018.04.009 (2018) 